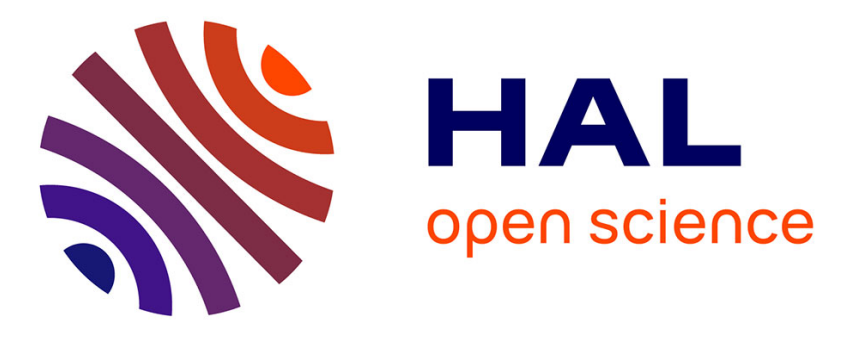

\title{
Tunable terahertz response of split ring resonator loaded along planar Goubau line
}

\author{
Abdallah Chhahadih, Mohamad Faran, Abbas Ghaddar, Gabriel Moreno, \\ Youssef Haidar, Tahsin Akalin
}

\section{- To cite this version:}

Abdallah Chhahadih, Mohamad Faran, Abbas Ghaddar, Gabriel Moreno, Youssef Haidar, et al.. Tunable terahertz response of split ring resonator loaded along planar Goubau line. 2019 IEEE 19th Mediterranean Microwave Symposium (MMS), Oct 2019, Hammamet, Tunisia. 9157259, 2 p., 10.1109/MMS48040.2019.9157259 . hal-03329004

\section{HAL Id: hal-03329004 https://hal.science/hal-03329004}

Submitted on 30 Aug 2021

HAL is a multi-disciplinary open access archive for the deposit and dissemination of scientific research documents, whether they are published or not. The documents may come from teaching and research institutions in France or abroad, or from public or private research centers.
L'archive ouverte pluridisciplinaire HAL, est destinée au dépôt et à la diffusion de documents scientifiques de niveau recherche, publiés ou non, émanant des établissements d'enseignement et de recherche français ou étrangers, des laboratoires publics ou privés. 


\section{Tunable Terahertz Response of Split Ring Resonator Loaded along Planar Goubau Line}

\author{
Abdallah Chhahadih \\ Faculty of science, section $V$, Lebanese \\ University, Nabatieh Lebanon
}

Mohamad Faran

Faculty of science, section $V$, Lebanese

University, Nabatieh, Lebanon

\author{
Abbas Ghaddar \\ Faculty of science, section $V$, Lebanese \\ University Nabatieh Lebanon \\ Gabriel Moreno, \\ Institute of Electronic, \\ Microelectronic and Nanotechnology \\ (IEMN), Lille 1 University, France.
}

\author{
Youssef Haidar \\ Faculty of science, section $V$, Lebanese \\ University, Nabatieh, Lebanon \\ Tahsin Akalin, \\ Institute of Electronic, \\ Microelectronic and Nanotechnology \\ (IEMN), Lille 1 University, France.
}

\begin{abstract}
In this communication, a computational and experimental investigation at terahertz frequencies of Planar Gaubau Line combined with Split Ring Resonator is reported. Different parameters such as gap width, position and gap orientation that tuned the resonance peak are studied. The obtained results are useful for the understanding of coupling mechanism between a radially polarized wave along a metallic strip and a Split Ring Resonator.
\end{abstract}

Keywords-Split Ring Resonator, Terahertz, VNA Characterization, Planar Goubau Line

\section{INTRODUCTION}

Metamaterials (MMs) have increasingly attracted huge attention thanks to their exceptional electromagnetic properties such as negative permittivity, negative permeability and negative refractive index media [1]. Researchers have demonstrated various $\mathrm{THz}$ devices for different applications, including imaging, sensing, filtering, superlenses, and antennas for security and communication systems [1-7].

Resonant-type MMs like Split Ring Resonator (SRR) has been widely used due to the inherent resonance characteristics present in such MM structures that result in a sharp cut-off around the resonance frequency $[1,2,4]$. The characteristic of SRR resonance depends on different parameters such as, size, shape, gap width'etc...[1,2, 4]. In this communication we present a study about the parameters that influence the resonance response of a SRR loaded along Planar Gaubau Line (PGL). We have focused on the influence orientation of the gap position with respect to the PGL on the resonance peak.

\section{FABRICATION AND CHARACTERISATION}

All the fabrication processes have been carried under the same conditions using E-beam lithography, with identical dimensions to those of the designs. The characterization of PGL structures requires coplanar wave (CPW) pads. To perform on-wafer measurements, a transition from the excitation access to the PGL line has been studied. The geometry of the CPW-PGL transition, designed on HR-Si substrate has been optimized using commercial full-wave EM simulation software MWS-CST (metals conductivity of $7 \times 10^{6} \mathrm{~S} \mathrm{~m}^{-1}$ and permittivity of the Si substrate is 11.7 ) and measured using Vector Network Analyzer (VNA). They are realized with Agilent $8510 \mathrm{XF}$ VNA from 0 to $0.110 \mathrm{THz}$ range and Anritsu 37147C VNA associated with a frequency multiplier to work on $0.14-0.22 \mathrm{THz}$ and $0.22-0.325 \mathrm{THz}$ range.

\section{EXPERIMENTAL RESULTS AND DISCUSSION}

Simulation and experimental studies of different parameters have been investigated such as gap size or gap orientation of SRR loaded along PGL. Figure 1 presents a fabrication image of PGLs loaded with rotated SRRs with different angles. The introduced designs have been fabricated on HR-Si $(380 \mu \mathrm{m})$ and then thinned mechanically down to $100 \mu \mathrm{m}$. The PGL dimension is $4-\mathrm{mm}$-long with a $5-\mu \mathrm{m}$ width made of $\mathrm{Ti} / \mathrm{Au}$ $(50 / 450 \mathrm{~nm})$. All SRR structures have circular shapes with a radius of $50 \mu \mathrm{m}$ and gap width of $5 \mu \mathrm{m}$.

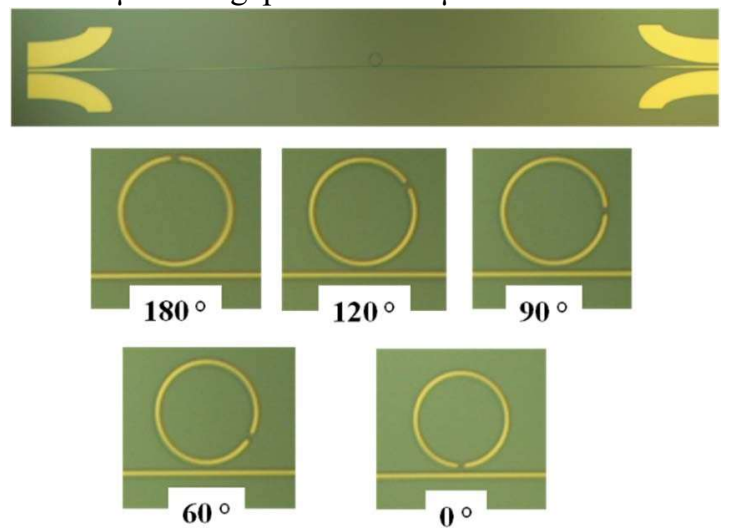

Fig. 1: SRRs loaded on PGL with different angles

PGL-design is optimized in order to obtain a broadband electromagnetic spectrum from 0 to $600 \mathrm{GHz}$. Figure 2 presents the simulation spectrum with frequencies up to 600 $\mathrm{GHz}$ (Figure $2 \mathrm{~A}$ ) and measurement curve with frequencies up to $325 \mathrm{GHz}$. 

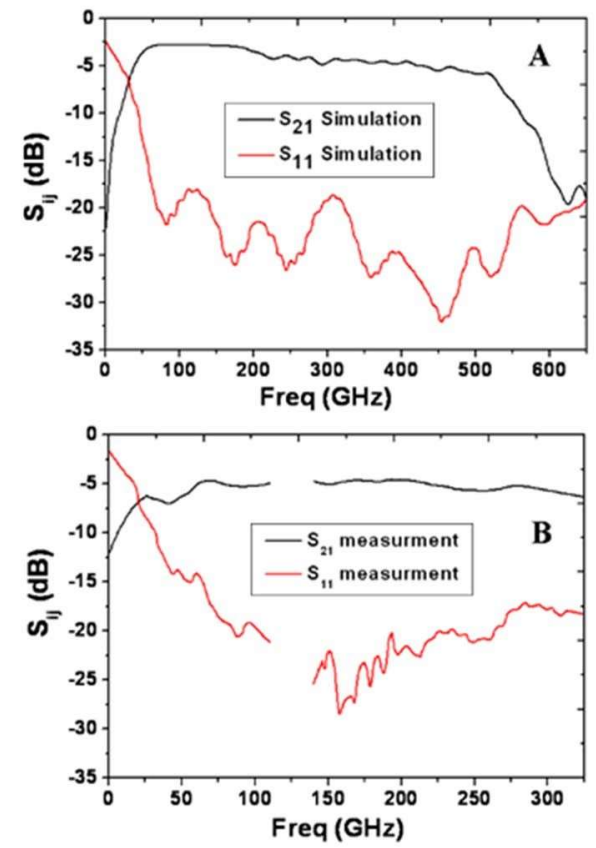

Fig. 2. S-parameters of simulation spectra of the introduced PGL

Regarding the simulation spectrum and for frequencies lower than $500 \mathrm{GHz}$, the transmission loss is approximately $-4.5 \mathrm{~dB}$ and the loss level is roughly the same along $0-500 \mathrm{GHz}$ band. A cut off frequency of about $500 \mathrm{GHz}$ has been observed. This cut-off frequency is due to substrate-mode propagation in the HR-Si substrate instead of Goubau-mode propagation. The thickness of substrate influences the cut-off frequency of the propagating substrate modes. Decreasing substrate thickness allows an increase in the cut-off frequency of the substrate modes $[4,8]$. The transmission loss of the measured PGL is about $-5 \mathrm{~dB}$. This value can be controlled by adjusting certain parameters such as: width and length of the PGL and the substrate medium. More details about the transition and the PGL are discussed elsewhere $[4,8]$

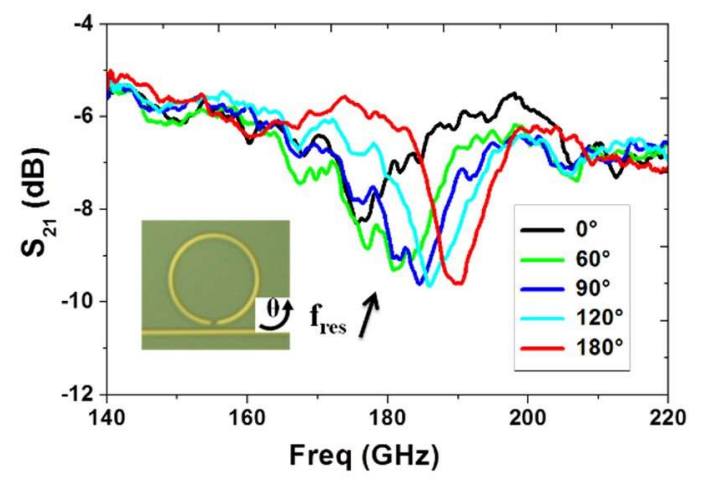

Fig. 3. Transmission spectra of the introduced structures shown in Fig.1.

Figure 3 presents the measurements of the different PGLs loaded with different SRR. By comparing the $\mathrm{S}_{21}$ parameters, one can note that the resonance frequency is shifted toward the high frequencies as increasing the rotation angle. Such kind of structure could be used to design a mm-wave filters.

\section{CONCLUSION}

Planar goubau line loaded with split ring resonators is presented. The influence of the orientation of the gap of the is presented. The results show that the resonance is not only due to SRR dimensions. More extensive simulations and characterizations will be presented in the final paper in order to provide further information about the influence of the orientation of the gap with respect to the PGL. In addition, we present in the final paper a passive tuning of SRRs response accomplished by shifting their location w.r.t the center of the PGL. The position of SRR along the transmission line can be controlled to attain maximum resonance.

\section{REFERENCES}

[1] W.C. Chen et al., "Controlling Gigahertz and Terahertz Surface Electromagnetic Waves with Metamaterial Resonators," Phys. Rev. X vol 1, 021016, 2011.

[2] A. Horestani et al, "Metamaterial-Inspired Bandpass Filters for Terahertz Surface Waves on Goubau Lines," IEEE-Terahertz Science and Technology Journal, vol 3, 740-747, 2013

[3] A. Chahadih et al., "Low Loss Transitions and Microstrip Lines on Cyclo-Olefin Co-Polymer Substrate for Terahertz Applications," IEEE, MMS-Lebanon, 02-05 September 2013.

[4] T. Aklain, "Terahertz Filters based on Planar Goubau Transmission Lines with Multi Split Ring Resonators," IEEE-IRMMW THz Mainz, Germany -02-06, 2013.

[5] D. Hu et al., "Dispersion characteristic of ultrathin terahertz planar lenses based on metasurface. Opt. Commun," vol 322, 164-168, 2014. [6]. M. Beruete et al., "Terahertz Corrugated and Bull's-Eye Antennas IEEETerahertz Science and Technology Journal", vol 3, 851-858, 2013. [7] A. Chahadih et al., "Planar Goubau Lines for on chip terahertz microscopy, IEEE-IRMMW THz", Tucson, Az, USA, 14-19 September 2014.

[8]. M. Zehar et al., "Low Loss Terahertz Planar Goubau Line on High Resistivity Silicon Substrate," IEEE, MMS-Lebanon, 02-05 September 2013. 